\title{
DIRECT CURRENT ELECTRIC POTENTIAL IN AN ANISOTROPIC HALF-SPACE WITH VERTICAL CONTACT CONTAINING A CONDUCTIVE 3D BODY
}

\author{
PING LI AND FRANK STAGNITTI
}

Received 17 January 2002

Detailed studies of anomalous conductors in otherwise homogeneous media have been modelled. Vertical contacts form common geometries in galvanic studies when describing geological formations with different electrical conductivities on either side. However, previous studies of vertical discontinuities have been mainly concerned with isotropic environments. In this paper, we deal with the effect on the electric potentials, such as mise-à-la-masse anomalies, due to a conductor near a vertical contact between two anisotropic regions. We also demonstrate the interactive effects when the conductive body is placed across the vertical contact. This problem is normally very difficult to solve by the traditional numerical methods. The integral equations for the electric potential in anisotropic half-spaces are established. Green's function is obtained using the reflection and transmission image method in which five images are needed to fit the boundary conditions on the vertical interface and the air-earth surface. The effects of the anisotropy of the environments and the conductive body on the electric potential are illustrated with the aid of several numerical examples.

\section{Introduction}

In spite of the fact that real media are strongly influenced by the directional and spatial variabilities of conductivity or resistivity, the attention of researchers has mainly been directed to problems in which purely isotropic, inhomogeneous isotropic, or homogeneous anisotropic media contain conductive bodies. The more general problems of inhomogeneous anisotropic media containing conductive bodies have not yet been addressed.

Asten [1] obtained an analytical solution for the potential due to a point source in a homogeneous anisotropic half-space, where one of the principal axes of the conductivity tensor is parallel to the ground surface. Pal and Dasgupta [16] also studied a special case of the electrical potential in an inhomogeneous anisotropic half-space. An extension of this was made by Pal and Mukherjee [17] who considered a two-layered conducting earth with dipping anisotropy. Negi and Saraf [15] discussed layer anisotropy problems in detail, and they gave the potential due to a point source in a variety of cases. All of these studies do not address the problem of anomalous perturbing bodies within the anisotropic medium. 


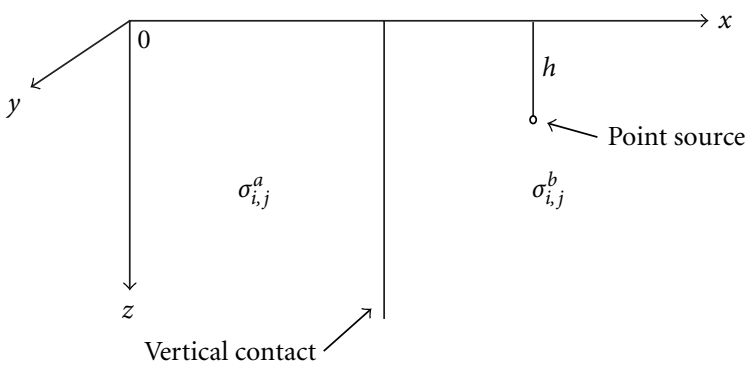

Figure 2.1. A point source embedded in an anisotropic half-space with vertical contact.

Tabarovskii $[18,19]$ presented a pair of integral equations for calculating direct current anomalies in an anisotropic medium. However, Tabarovskii $[18,19]$ did not present any numerical applications and the solution was only suitable for the simple case where the conductivity tensor had nonzero terms on the diagonal only. Eloranta [2] dealt with the mise-à-la-masse method, applied to study the effect on apparent resistivity due to perfect conductors in an anisotropic half-space. But the limitations of this study are that the halfspace is a homogeneous medium and one of the principal axes of the conductivity tensor is parallel to the earth's surface.

Eskola and Hongisto [6] attempted to take into account anisotropic electrical conductivity in an integral equation formulation. Their formulation however proved to be erroneous due to an incorrect Green's function in the integral equation (Eskola [3]). Eloranta [2] studied the modelling of mise-à-la-masse anomalies for geophysical surveying based on an integral equation. Eskola et al. [8] used an integral equation for the modelling of the electrical potential in and around a thin conductor. Eskola and Hongisto [7], based on the works of Eskola [4], Xiong et al. [20], and Tabarovskii [18, 19], presented mathematical modelling of the resistivity and IP effects of an anisotropic body in an isotropic environment using the boundary integral equation method.

Flykt et al. [9] gave an integral equation for an anisotropic spherical body in a homogeneous anisotropic environment and calculated the responses when the body was embedded in a uniaxially anisotropic environment.

Li and Uren [13], using the Green's function given by Li and Uren [14] and the integral equation given by Eskola [5], simulated the electrical potential in arbitrarily homogeneous anisotropic half-space containing a conductive body.

The purpose of this paper is to show the behavior of the direct current electric potential arising from a point source in a 3D perfect conductor in an anisotropic half-space with a vertical contact. The integral equation method is employed.

\section{The definition of the problem}

2.1. The physical model of the problem. The physical model is illustrated in Figure 2.1. The point source and conductive body may be located anywhere in the half-space. However, for convenience, the vertical contact is located in the plane $x=0$ which extends to infinity in the positive and negative $y$-directions and the positive $z$-direction. The 
conductivities of the media on either side of the contact are $\sigma_{i, j}^{a}$ and $\sigma_{i, j}^{b}$, where $i$ and $j$ are from 1 to 3 which indicate the three coordinate directions.

2.2. Mathematical formulation of the problem. First consider a point source located in the half-space. The problem can be formulated mathematically by the following boundary value problems:

$$
\begin{gathered}
\nabla \cdot \sigma_{i, j}^{a} \cdot \nabla u_{a}(\mathbf{R})=I \delta\left(\mathbf{R}-\mathbf{R}^{p}\right), \\
\nabla \cdot \sigma_{i, j}^{b} \cdot \nabla u_{b}(\mathbf{R})=I \delta\left(\mathbf{R}-\mathbf{R}^{p}\right),
\end{gathered}
$$

where $\mathbf{R}(x, y, z)$ is the position of any point in the medium, $\mathbf{R}^{P}\left(x^{p}, y^{p}, z^{p}\right)$ is the position of the direct current source, $u_{a}(\mathbf{R})$ and $u_{b}(\mathbf{R})$ are the potentials due to the source at any point $\mathbf{R}$ in media $a$ and $b$, respectively, $\delta$ is the Dirac delta function, and $I$ is the electrode current.

The governing partial differential equation is subject to the following boundary conditions:

(a) on the air-earth interface, the normal component of the current density must be zero. For $x \geq 0$,

$$
\mathbf{n}_{z} \cdot \sigma_{i, j}^{a} \cdot \nabla u_{a}(\mathbf{R})=0
$$

and for $x<0$,

$$
\mathbf{n}_{z} \cdot \sigma_{i, j}^{b} \cdot \nabla u_{b}(\mathbf{R})=0
$$

(b) on the interface between the two media, the potential densities must be equal:

$$
u_{a}(\mathbf{R})=u_{b}(\mathbf{R})
$$

(c) on the interface between the two media, the current densities must be equal too:

$$
\mathbf{n}_{x} \cdot \sigma_{i, j}^{a} \cdot \nabla u_{a}(\mathbf{R})=\mathbf{n}_{x} \cdot \sigma_{i, j}^{b} \cdot \nabla u_{b}(\mathbf{R}),
$$

the boundary conditions (b) and (c) are called the continuous conditions;

(d) when any one of $\pm x, \pm y$, or $z \rightarrow \infty$, the direct current point source potential must reduce to zero:

$$
u_{a}(\mathbf{R} \longrightarrow \infty) \longrightarrow 0, \quad u_{b}(\mathbf{R} \longrightarrow \infty) \longrightarrow 0
$$

Now consider a conductive body in the half-space with a point source as depicted in Figure 2.2. The potential governing equations for an anisotropic medium in the Cartesian coordinate system are

$$
\begin{aligned}
& \nabla \cdot \sigma_{i, j}^{a} \cdot \nabla u_{a}(\mathbf{R})=-\frac{\sigma\left(\mathbf{R}_{0}\right)}{\epsilon_{0}} \delta\left(\mathbf{R}-\mathbf{R}_{0}\right)+I \delta\left(\mathbf{R}-\mathbf{R}^{p}\right), \\
& \nabla \cdot \sigma_{i, j}^{b} \cdot \nabla u_{b}(\mathbf{R})=-\frac{\sigma\left(\mathbf{R}_{0}\right)}{\epsilon_{0}} \delta\left(\mathbf{R}-\mathbf{R}_{0}\right)+I \delta\left(\mathbf{R}-\mathbf{R}^{p}\right),
\end{aligned}
$$




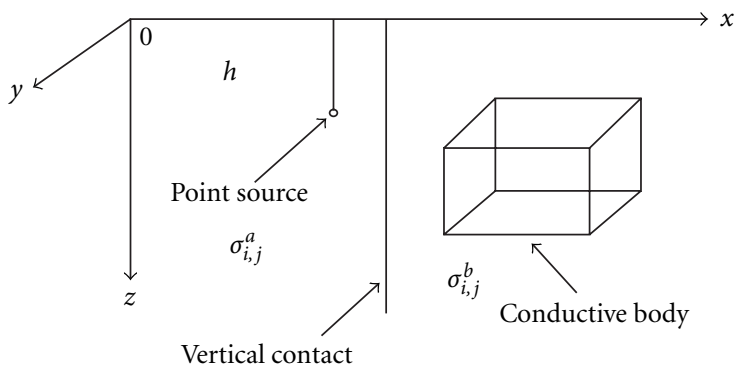

Figure 2.2. A conductive body and a point source embedded in an anisotropic half-space with vertical contact. The body can be of any shape.

where $\sigma\left(\mathbf{R}_{0}\right)$ is the induced charge density on the surface of the body, $u_{a}(\mathbf{R})$ and $u_{b}(\mathbf{R})$ are the potentials in the host media due to the induced surface charge density on the body and primary point source function $I \delta\left(\mathbf{R}-\mathbf{R}^{p}\right)$, and $\mathbf{R}_{0}\left(x_{0}, y_{0}, z_{0}\right)$ is the source position on the surface of the body of the induced charge density.

As the potential in this system results from a point source, the expressions of the potentials are different when the source location is in different media $a$ and $b$. In other words, the potentials $u_{a}$ and $u_{b}$ have different expressions in media $a$ and $b$. Therefore, for mathematical convenience, a new variable $U$ is introduced to express the potentials when the point source is located in different media. That is

$$
U(\mathbf{R})= \begin{cases}u_{a a}(\mathbf{R}), & \text { if } x>0, x_{0}>0 \\ u_{a b}(\mathbf{R}), & \text { if } x>0, x_{0} \leq 0 \\ u_{b b}(\mathbf{R}), & \text { if } x \leq 0, x_{0} \leq 0 \\ u_{b a}(\mathbf{R}), & \text { if } x \leq 0, x_{0}>0\end{cases}
$$

Using theories of Green's function and integral equation methods, we can in this case transform the boundary value problem into an integral equation given by

$$
U(\mathbf{R})=a\left(\int_{S} G\left(\mathbf{R}, \mathbf{R}_{0}\right) f_{n}\left(\mathbf{R}_{0}\right) d S_{0}+G\left(\mathbf{R}, \mathbf{R}^{p}\right) I\left(\mathbf{R}^{p}\right)\right),
$$

where $G\left(\mathbf{R}, \mathbf{R}_{0}\right)$ is the appropriate Green's function of the half-space presented in detail in Section 3 and $f_{n}\left(\mathbf{R}_{0}\right)$ is the unknown normal component of current density on the surface of the conductive body.

Equation (2.9) is a Fredholm integral equation of the first kind. In the perfectly conductive anomalous body, the total potential is a constant $\left(U_{c}\right)$. Its value can be determined from the following equations:

$$
\begin{gathered}
U_{c}(\mathbf{R})=\int_{S} G\left(\mathbf{R}, \mathbf{R}_{0}\right) f_{n}\left(\mathbf{R}_{0}\right) d S_{0}+G\left(\mathbf{R}, \mathbf{R}^{p}\right) I\left(\mathbf{R}^{p}\right), \\
\int f_{n} d S=I,
\end{gathered}
$$


for surface $S$ enclosing a source-emitting current $I$, and

$$
\int f_{n} d S=0
$$

for surface $S$ which does not enclose current sources. The $\mathbf{R}_{0}$ lies on the surface of the body. From (2.9), (2.10), (2.11), and (2.12), if the appropriate Green's function is known, there the current density $f_{n}\left(\mathbf{R}_{0}\right)$ and the constant potential $U_{c}$ can be obtained.

\section{Green's function}

Based on the theory of the reflection and transmission image (Li and Uren [11]), five images of the primary source were determined for fitting the boundary conditions presented in Section 2. The concept model of the image positions is illustrated in Figure 3.1. The correspondent Green's functions are as follows.

Case 1. When the point source is located in medium $a$,

$$
U_{a a}=\frac{I_{0}}{4 \pi} \sum_{i=1}^{4} \frac{A_{i}}{\eta_{i}^{a}}
$$

when the observation point is in medium $a$, where

$$
\eta_{i}^{a}=\sqrt{\sigma_{1,1}^{a} X_{i}^{2}+\sigma_{2,2}^{a} Y_{i}^{2}+\sigma_{3,3}^{a} Z_{i}^{2}+2 \sigma_{1,2}^{a} X_{i} Y_{i}+2 \sigma_{1,3}^{a} X_{i} Z_{i}+2 \sigma_{2,3}^{a} Y_{i} Z_{i}}
$$

is called the weighted distance from the source point or the images point of the source to the observation point in the half-space, and

$$
U_{a b}=\frac{I_{0}}{4 \pi} \sum_{i=5}^{6} \frac{A_{i}}{\eta_{i}^{b}}
$$

when the observation point is in medium $b$, where

$$
\eta_{i}^{b}=\sqrt{\sigma_{1,1}^{b} X_{i}^{2}+\sigma_{2,2}^{b} Y_{i}^{2}+\sigma_{3,3}^{b} Z_{i}^{2}+2 \sigma_{1,2}^{b} X_{i} Y_{i}+2 \sigma_{1,3}^{b} X_{i} Z_{i}+2 \sigma_{2,3}^{b} Y_{i} Z_{i}}
$$

and where $X_{i}=x-x_{i}, Y_{i}=y-y_{i}$, and $Z_{i}=z-z_{i}$.

Case 2. When the point source is located in medium $b$,

$$
U_{b a}=\frac{I_{0}}{4 \pi} \sum_{i=5}^{6} \frac{B_{i}}{\eta_{i}^{a}}
$$

when the observation point is in medium $a$, and

$$
U_{b b}=\frac{I_{0}}{4 \pi} \sum_{i=1}^{4} \frac{B_{i}}{\eta_{i}^{b}}
$$

when the observation point is in medium $b$. 
(RTP) Reflection image of TP

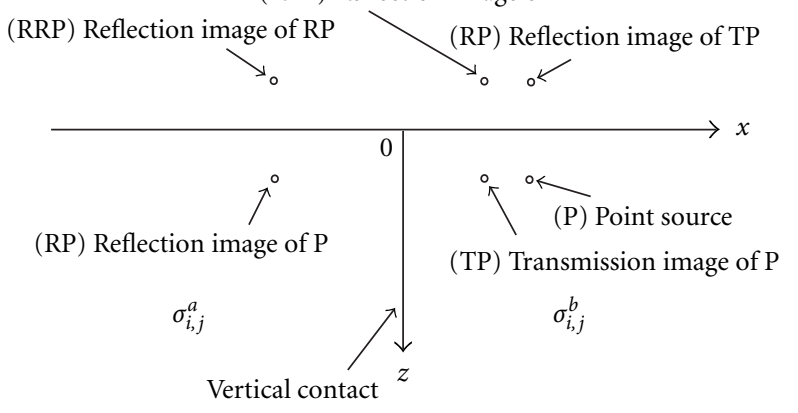

Figure 3.1. Image positions of a point source that is located in an anisotropic half-space with vertical contact.

Table 3.1 summarizes the image-source positions and their potential coefficients when the anisotropic method of images is applied for each of the planar interfaces in the model ( $\mathrm{Li}$ and Uren $[10,11,12]): P_{1}\left(x_{1}, y_{1}, z_{1}\right)$ is the point source position and $P_{2}\left(x_{2}, y_{2}, z_{2}\right)$ is its vertical contact interface reflection image position; $P_{3}\left(x_{3}, y_{3}, z_{3}\right)$ and $P_{4}\left(x_{4}, y_{4}, z_{4}\right)$ are the air-earth surface reflection image locations of $P_{1}$ and $P_{2} ; P_{5}\left(x_{5}, y_{5}, z_{5}\right)$ is the transmission image position of point source $P_{1}\left(x_{1}, y_{1}, z_{1}\right) ; P_{6}\left(x_{6}, y_{6}, z_{6}\right)$ is the air-earth surface reflection image position of $P_{5}$; and $A_{i}$ and $B_{i}(i=1,2, \ldots, 6)$ are the corresponding reflection and transmission coefficients. They depend on the vertical interface and air-earth surface boundary conditions as stated above. See columns 2 and 4 of Table 3.1.

In the table,

$$
\begin{aligned}
& A_{2}=\frac{I^{[a]}-I^{[b]} \mathcal{S}^{[b]}}{I^{[a]}+I^{[b]} \mathcal{S}^{[b]}}, \\
& B_{2}=\frac{I^{[b]}-I^{[a]} \mathcal{S}^{[a]}}{I^{[b]}+I^{[a]} \mathcal{S}^{[a]}}, \\
& A_{5}=\frac{2\left(I^{[a]}\right)^{2}}{I^{[b]} I^{[a]}+\left(I^{[b]}\right)^{2} \mathcal{S}^{[b]}}, \\
& B_{5}=\frac{2\left(I^{[b]}\right)^{2}}{I^{[a]} I^{[b]}+\left(I^{[a]}\right)^{2} s^{[a]}} .
\end{aligned}
$$

\section{The numerical solution to the integral equation}

By dividing the surface $S$ (containing the conductive body) into so small subareas $\Delta s_{k}$ that the normal component of the current density can be taken as a constant within each subarea, and requiring that (2.10) holds at the centre of each subarea, the following set of linear algebraic equations is obtained:

$$
\mathbf{A} \cdot \mathbf{F}=\mathbf{B}
$$


Table 3.1. Image source positions and their potential coefficients.

\begin{tabular}{|c|c|c|c|c|}
\hline \multicolumn{2}{|c|}{ Case 1} & \multicolumn{2}{|c|}{ Case 2} & \multirow{2}{*}{ Source name } \\
\hline Image point & Coefficients & Image point & Coefficients & \\
\hline $\begin{array}{l}x_{1}=x^{p} \\
y_{1}=y^{p} \\
z_{1}=z_{0}^{p}\end{array}$ & $A_{1}=1$ & $\begin{array}{l}x_{1}=x^{p} \\
y_{1}=y^{p} \\
z_{1}=z_{0}^{p}\end{array}$ & $B_{1}=1$ & $\begin{array}{l}P_{1}: \text { initial } \\
\text { point } \\
\text { source }\end{array}$ \\
\hline $\begin{array}{l}x_{2}=-x_{1} \\
y_{2}=2 r_{y}^{[a]} x_{1}+y_{1} \\
z_{2}=2 r_{z}^{[a]} x_{1}+z_{1}\end{array}$ & $A_{2}(\operatorname{see}(3.7))$ & $\begin{array}{l}x_{2}=-x_{1} \\
y_{2}=2 r_{y}^{[b]} x_{1}+y_{1} \\
z_{2}=2 r_{z}^{[b]} x_{1}+z_{1}\end{array}$ & $B_{2}(\operatorname{see}(3.8))$ & $\begin{array}{l}P_{2}: \text { vertical } \\
\text { reflection } \\
\text { image of } P_{1}\end{array}$ \\
\hline $\begin{array}{l}x_{3}=2 r_{x}^{[a]} z_{1}+x_{1} \\
y_{3}=2 r_{y}^{[a]} z_{1}+y_{1} \\
z_{3}=-z_{1}\end{array}$ & $A_{3}=A_{1}$ & $\begin{array}{l}x_{3}=2 r_{x}^{[b]} z_{1}+x_{1} \\
y_{3}=2 r_{y}^{[a]} z_{1}+y_{1} \\
z_{3}=-z_{1}\end{array}$ & $B_{3}=B_{1}$ & $\begin{array}{l}P_{3}: \text { surface } \\
\text { reflection } \\
\text { image of } P_{1}\end{array}$ \\
\hline $\begin{array}{l}x_{4}=2 r_{x}^{[b]} z_{2}+x_{2} \\
y_{4}=2 r_{y}^{[b]} z_{2}+y_{2} \\
z_{4}=-z_{2}\end{array}$ & $A_{4}=A_{2}$ & $\begin{array}{l}x_{4}=2 r_{x}^{[a]} z_{2}+x_{2} \\
y_{4}=2 r_{y}^{[a]} z_{2}+y_{2} \\
z_{4}=-z_{2}\end{array}$ & $B_{4}=B_{2}$ & $\begin{array}{l}P_{4}: \text { surface } \\
\text { reflection } \\
\text { image of } P_{2}\end{array}$ \\
\hline $\begin{array}{l}x_{5}=-s x_{1} \\
y_{5}=r_{y}^{[a]} z_{1}-r_{y}^{[b]} x_{5} \\
z_{5}=r_{z}^{[a]} z_{1}-r_{z}^{[b]} x_{5}\end{array}$ & $A_{5}(\operatorname{see}(3.9))$ & $\begin{array}{l}x_{5}=-s x_{1} \\
y_{5}=r_{y}^{[b]} z_{1}-r_{y}^{[a]} x_{5} \\
z_{5}=r_{z}^{[b]} z_{1}-r_{z}^{[a]} x_{5}\end{array}$ & $B_{5}(\operatorname{see}(3.10))$ & $\begin{array}{l}P_{5}: \text { vertical } \\
\text { transmission } \\
\text { image of } P_{1}\end{array}$ \\
\hline $\begin{array}{l}x_{6}=2 r_{x}^{[b]} z_{5}+x_{2} \\
y_{6}=2 r_{y}^{[b]} z_{5}+y_{2} \\
z_{6}=-z_{5}\end{array}$ & $A_{6}=A_{5}$ & $\begin{array}{l}x_{6}=2 r_{x}^{[b]} z_{5}+x_{2} \\
y_{6}=2 r_{y}^{[a]} z_{5}+y_{2} \\
z_{6}=-z_{5}\end{array}$ & $B_{6}=B_{5}$ & $\begin{array}{l}P_{6}: \text { surface } \\
\text { reflection } \\
\text { image of } P_{5}\end{array}$ \\
\hline
\end{tabular}

where $\mathbf{A}$ is an $(M+1) \times(M+1)$ matrix:

$$
\mathbf{A}=\left(\begin{array}{ccccc}
\bar{G}_{1,1} & \bar{G}_{1,2} & \cdots & \bar{G}_{1, M} & -1.0 \\
\bar{G}_{2,1} & \bar{G}_{2,2} & \cdots & \bar{G}_{2, M} & -1.0 \\
\vdots & \vdots & \vdots & \vdots & \vdots \\
\bar{G}_{M, 1} & \bar{G}_{M, 2} & \cdots & \bar{G}_{M, M} & -1.0 \\
\Delta s_{1} & \Delta s_{2} & \cdots & \Delta s_{M} & 0.0
\end{array}\right)
$$

and $\mathbf{B}$ is the $(M+1)$ matrix $\left(G_{p 1}, G_{p 2}, \ldots, G_{p n}, I\right)$. When the point source is located outside of the body, the matrix becomes $\left(G_{p 1}, G_{p 2}, \ldots, G_{p n}, 0.0\right)$. However, for the mise-à-la-masse 
case, the matrix $\mathbf{B}$ is $(0,0.1, \ldots, 1.0)$. The unknown matrix is $\mathbf{F}=\left(f_{n}\left(\mathbf{R}_{01}\right), f_{n}\left(\mathbf{R}_{02}\right), \ldots\right.$, $\left.f_{n}\left(\mathbf{R}_{0 M}\right), U_{c}\right)$, where $U_{c}$ is the constant potential on the surface of the conductive body and

$$
\bar{G}_{i, k}=\int_{\Delta s_{k}} G\left(\mathbf{R}_{i}, \mathbf{R}_{0}\right) d S
$$

where $i, k=1,2, \ldots, M$. In (4.3), $i$ refers to the calculation subarea, $\mathbf{R}_{i}$ is the position of the centre of each $\Delta s_{i}$, and $k$ refers to the source subarea $\Delta s_{k} ; \mathbf{R}_{i}$ and $\mathbf{R}_{0}$ both run on the body surface. When the body contains the current source,

$$
\sum_{i=1}^{M} f_{n}\left(\mathbf{R}_{i}\right)=I
$$

and when the source is located outside of the body,

$$
\sum_{i=1}^{M} f_{n}\left(\mathbf{R}_{i}\right)=0
$$

The $M$ unknown current densities $f_{n}\left(\mathbf{R}_{i}\right)$ and the constant potential on the body can be calculated by solving the $(M+1)$ linear algebraic equations. Substitution of $f_{n}\left(\mathbf{R}_{i}\right)$ into the equation

$$
U(\mathbf{R})=a \sum_{i=1}^{M} f_{n}\left(\mathbf{R}_{i}\right) \bar{G}_{i, k}+a G\left(\mathbf{R}, \mathbf{R}^{p}\right) I\left(\mathbf{R}_{i}\right),
$$

which is obtained by discretization of the first term of (2.9), allows the calculation of the potential in the host medium due to the induced surface charge density on the body and the primary point source. When the point source is located outside of the body, the second term of this equation is zero.

The integrated Green's function defined by (4.3) converges when $i=k$ because of the $1 / \eta$ nature of $G$. This can be calculated using a one-dimensional analytical method followed by another multidimensional numerical approach when the body is represented by the cube-cell accumulation method, in which the cell surfaces are parallel to the Cartesian coordinate system (Li and Uren [13]).

\section{Numerical example}

Two examples are introduced in this paper for the demonstration of anisotropic effects in a vertical contrast half-space. The resistivity tensors in media $a$ and $b$ of the half-space for example 1 are

$$
\sigma_{i, j}^{1 a}=\left(\begin{array}{lll}
0.120 & 0.100 & 0.000 \\
0.100 & 0.420 & 0.000 \\
0.000 & 0.000 & 0.300
\end{array}\right), \quad \sigma_{i, j}^{1 b}=\left(\begin{array}{lll}
0.520 & 0.410 & 0.000 \\
0.410 & 0.420 & 0.000 \\
0.000 & 0.000 & 0.300
\end{array}\right),
$$


and for example 2 are

$$
\sigma_{i, j}^{2 a}=\left(\begin{array}{ccc}
0.3060 & 0.050 & 0.000 \\
0.050 & 0.210 & 0.000 \\
0.000 & 0.000 & 0.150
\end{array}\right), \quad \sigma_{i, j}^{2 b}=\left(\begin{array}{ccc}
0.150 & 0.105 & 0.000 \\
0.105 & 0.210 & 0.000 \\
0.000 & 0.000 & 0.150
\end{array}\right) .
$$

Their vertical contacts are at $x=0$. For mathematical convenience, a conductive rectangular prism $(6 \mathrm{~m} \times 6 \mathrm{~m} \times 8 \mathrm{~m})$ is located in various positions in this space. Using the linear equations (4.1) and (4.6), the equipotentials on either side of the contact can be determined. The cell surfaces are always parallel to the coordinate system as shown in Figure 2.2. For various positions of the body, the equipotential contours are given in Figures $5.1,5.2,5.3$, and 5.4 .

For example 1, Figure 5.1 shows the behavior of the potential arising from a point source and a perfectly conductive body when the source is located outside of the body. In Figure 5.1(a), we can clearly see the body profile when the observation plane is across the body. This is because the body is a perfect conductor and the body surface is as an equipotential surface. Figures 5.1(a), 5.1(b), and 5.1(c) illustrate equipotential contours when the observation plane is away from the body centre and Figures 5.1(d), 5.1(e), and 5.1(f) represent equipotential contours when the observation plane commences from the air-earth surface and closer to the body.

Figure 5.2 gives the equipotential contours for example 1 when the point source is located in the body (i.e., mise-à-la-masse). Figures 5.2(a), 5.2(b), and 5.2(c) show the equipotential contours when the observation plane from the body is away from the airearth surface. Figures 5.2(d), 5.2(e), and 5.2(f) show equipotential contours starting from the air-earth surface and away in $z$-direction.

Figures 5.3 and 5.4 represent equipotential contours for example 2 given by resistivity tensors defined by (5.2).

In Figure 5.3, the point source is located beside the conductive body. The interaction of the conductive body, the point source, and the vertical contact to the potentials are clearly shown in the equipotential contours of vertical and horizontal observation planes. Figure 5.4 shows the correspondent mise-à-la-masse case. The effects of the conductive body and the vertical contact are clearly demonstrated.

The figures also show the behavior of the potential arising from the perfectly conductive body and point source. We see that the equipotential contours are very much changed when the source is located closer to the body and vertical contact. This is because of the interaction between the charge densities on the vertical contact and the surface of the conductive body. At the interface between the two media, the equipotential contours are distorted. This indicates the existence of the charge which is reduced by the conductive body and the inhomogeneity of the media. When the conductive body is located on the left-hand side of the vertical contact, the potential in the right medium is dependent on the charge density on the contact, and the potential in the right-hand side is dependent on both surface charge densities of the conductive body and the vertical contact. However, when the conductive body is placed across the vertical contact, both media potentials are due to both charge densities. The implications for field work are clearly shown in our computational results. 


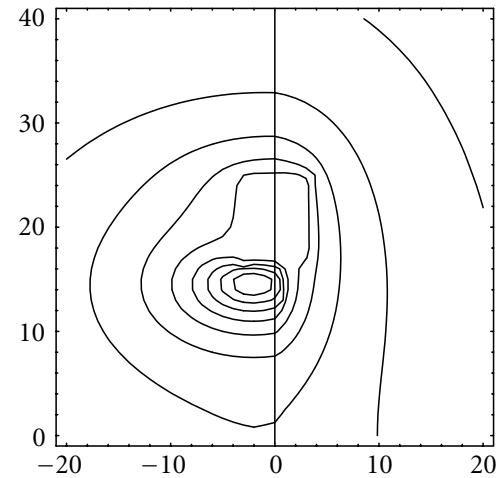

(a) Observation plane $y=2.5$.

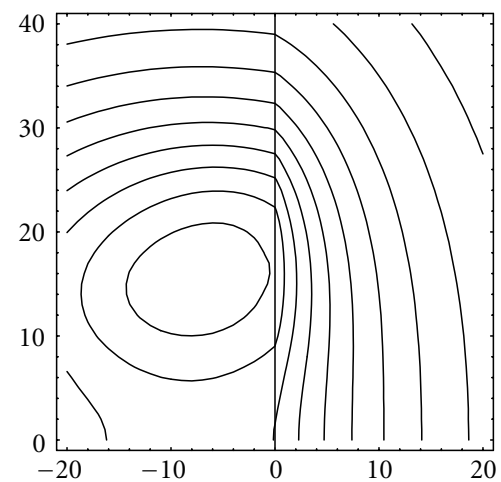

(c) Observation plane $y=10.0$.

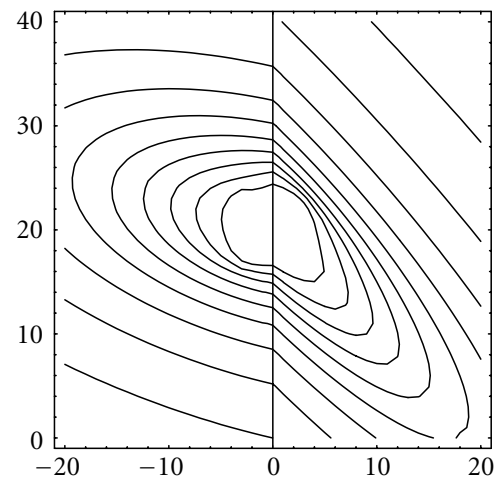

(e) Observation plane $z=25.0$.

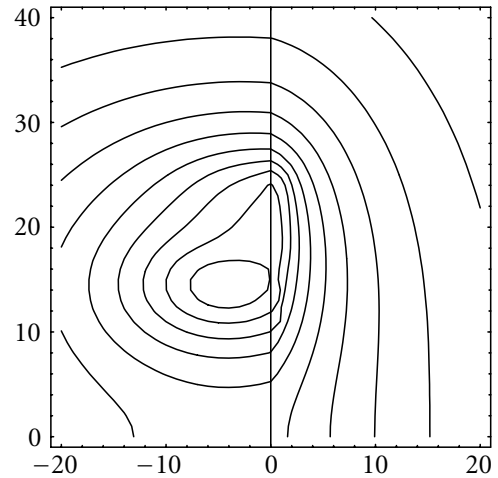

(b) Observation plane $y=5.0$.

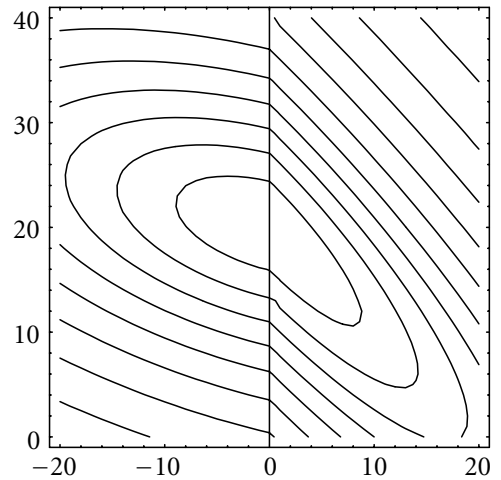

(d) Observation plane $z=0.0$.

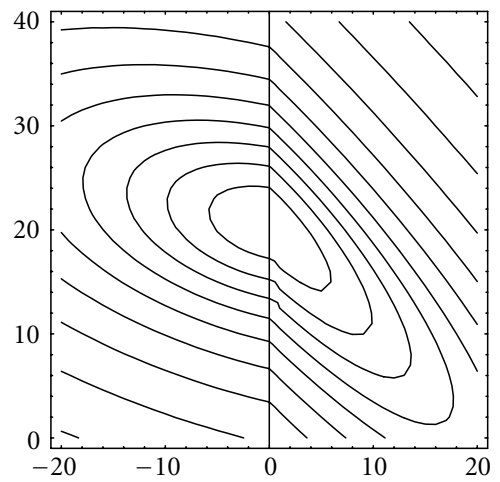

(f) Observation plane $z=30.0$.

Figure 5.1. Tensors $\sigma^{1 b}$ and $\sigma^{1 a}$ equipotential contours arising from a point source in an anisotropic medium in the presence of a conductive body. Figures 5.1(a), 5.1(b), and 5.1(c) show the equipotentials when the observation plane is $y=2.5,5.0$, and 10.0, respectively. Figures 5.1(d), 5.1(e), and 5.1 (f) show the equipotentials when the observation plane is $z=0.0,25.0$, and 30.0 , respectively. 


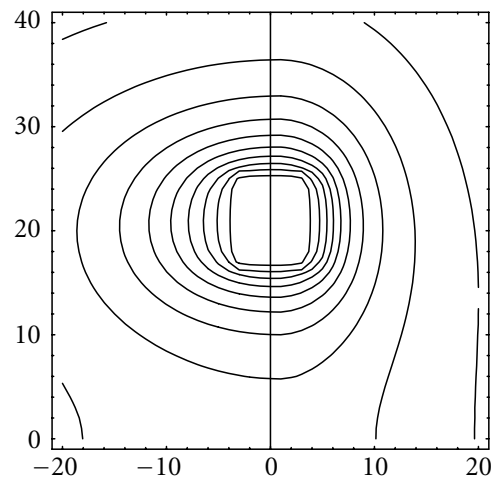

(a) Observation plane $y=2.5$.

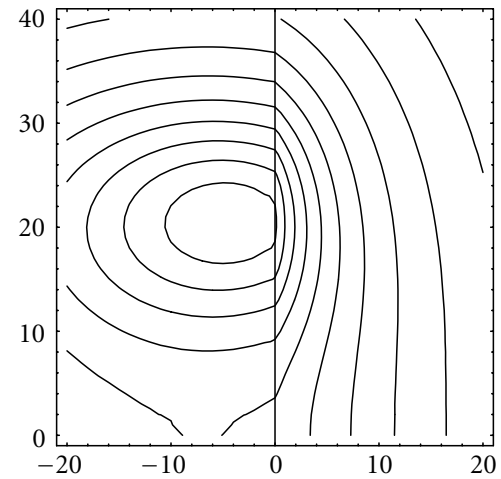

(c) Observation plane $y=10.0$.

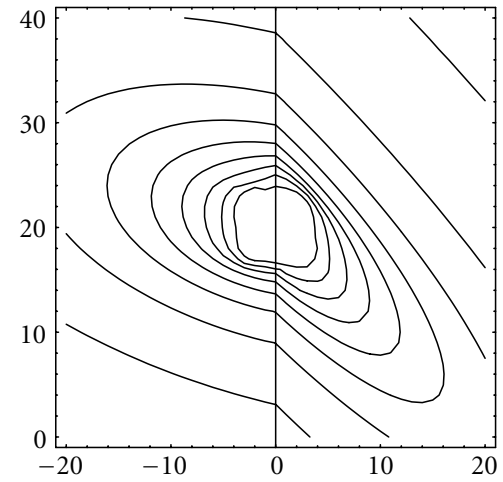

(e) Observation plane $z=25.0$.

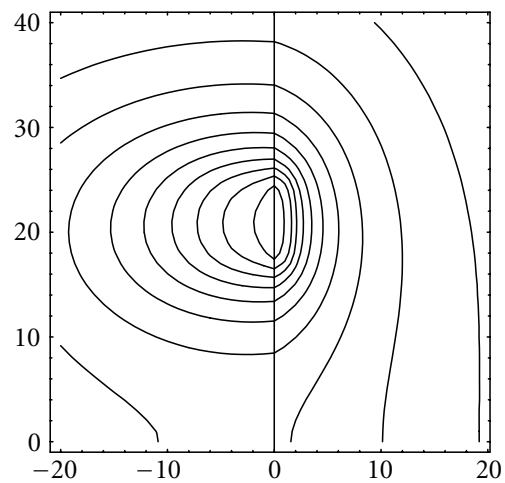

(b) Observation plane $y=5.0$.

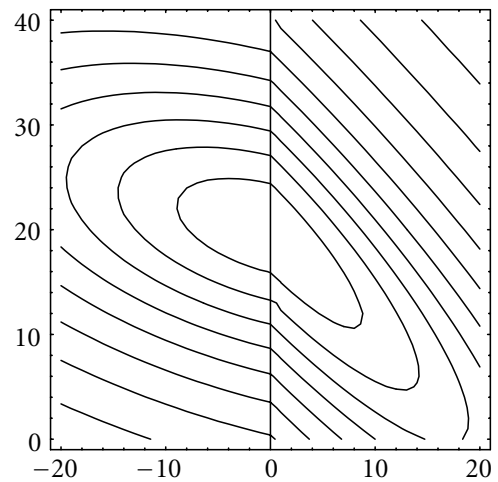

(d) Observation plane $z=0.0$.

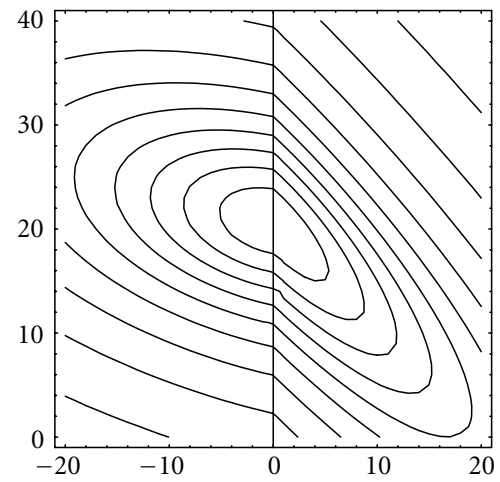

(f) Observation plane $z=30.0$.

Figure 5.2. Tensors $\sigma^{1 a}$ and $\sigma^{1 b}$ vertical and horizontal observation plane equipotential contours for the mise-à-la-masse potential arising from a conductive body. 


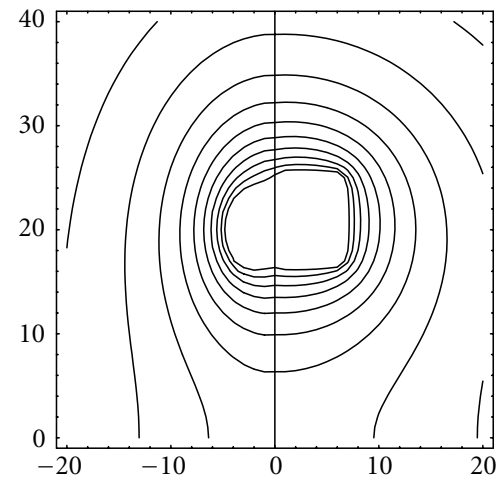

(a) Observation plane $y=2.5$.

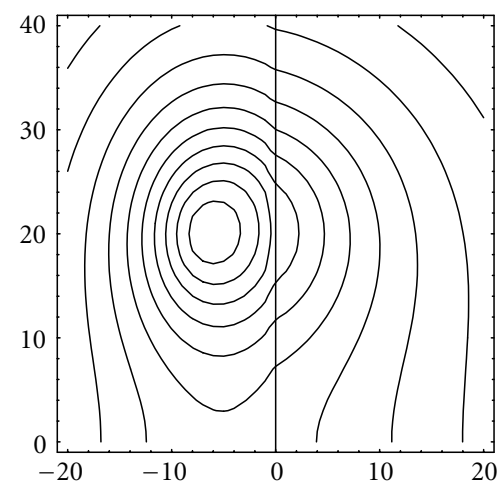

(c) Observation plane $y=10.0$.

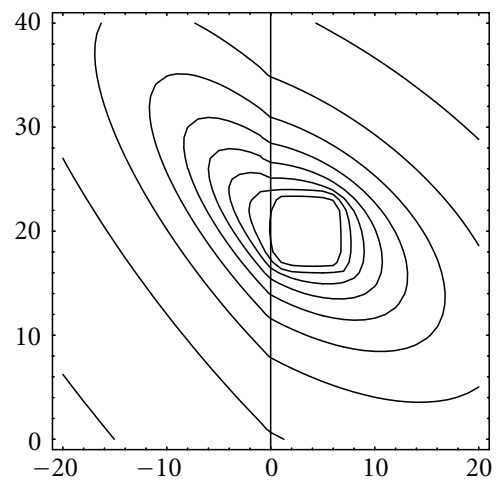

(e) Observation plane $z=25.0$.

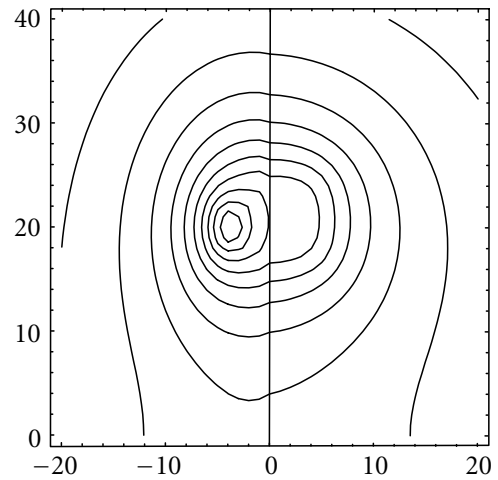

(b) Observation plane $y=5.0$.

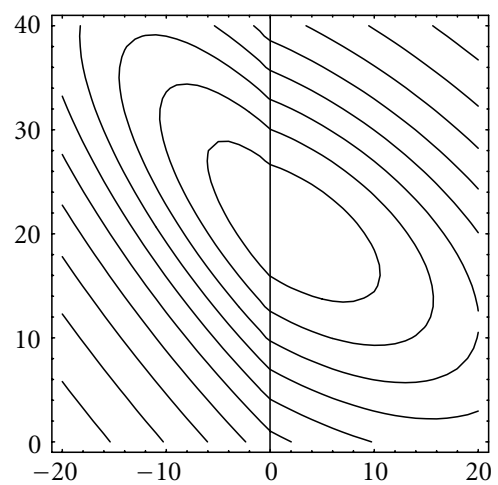

(d) Observation plane $z=0.0$.

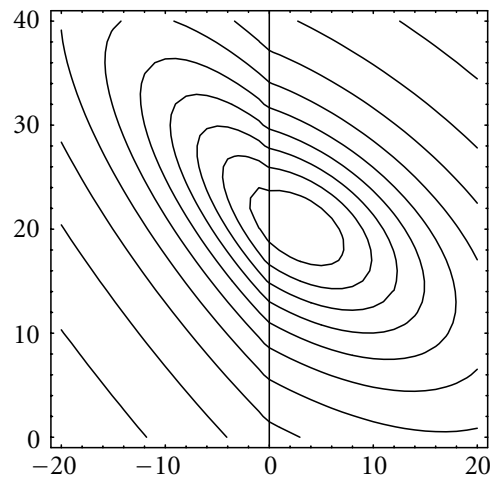

(f) Observation plane $z=30.0$.

Figure 5.3. Tensors $\sigma^{2 a}$ and $\sigma^{2 b}$ equipotential contours arising from a point source in an anisotropic medium with conductive body. Figures 5.3(a), 5.3(b), and 5.3(c) show the equipotentials when the observation plane is $y=2.5,5.0$, and 10.0, respectively. Figures 5.3(d), 5.3(e), and 5.3(f) show the equipotentials when the observation plane is $z=0.0,25.0$, and 30.0 , respectively. 


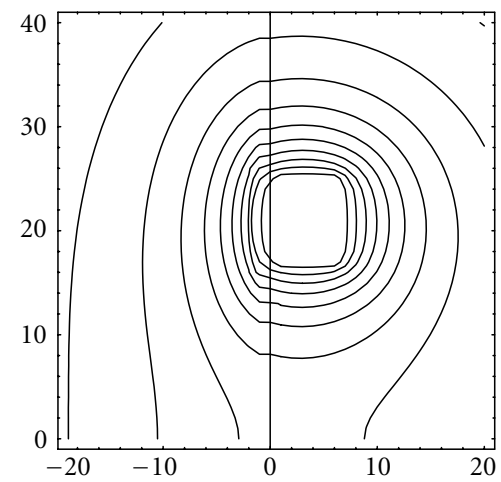

(a) Observation plane $y=2.5$.

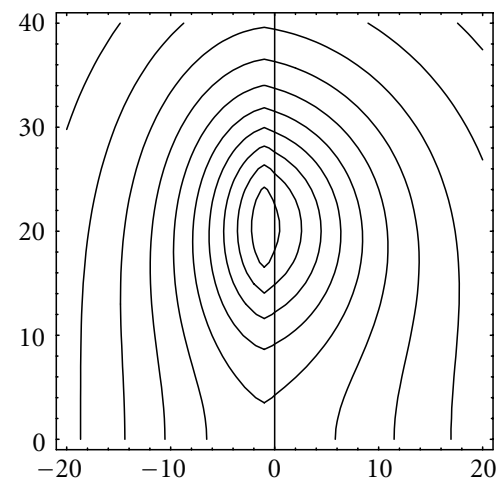

(c) Observation plane $y=10.0$.

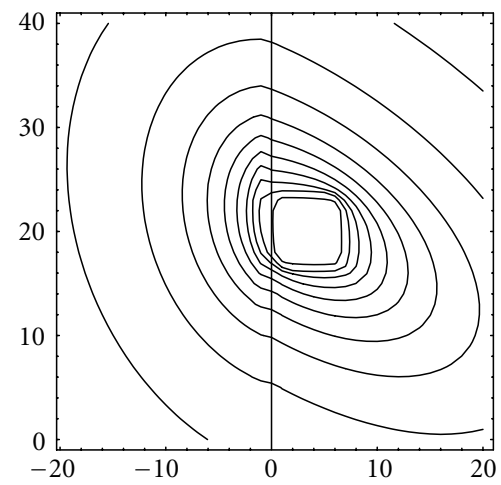

(e) Observation plane $z=25.0$.

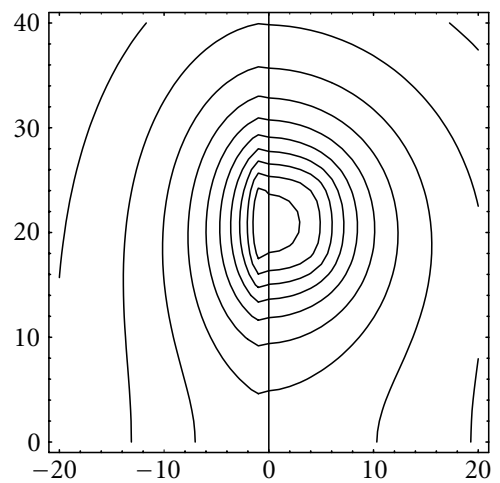

(b) Observation plane $y=5.0$.

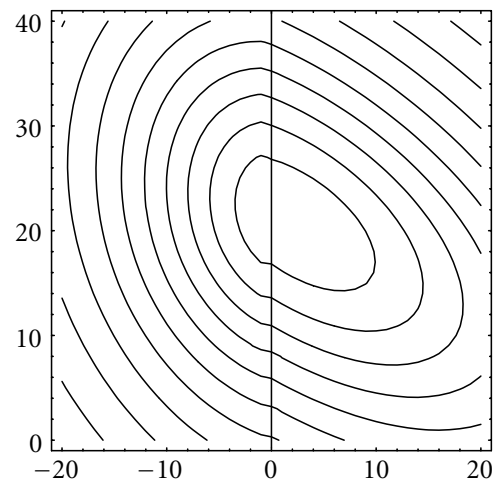

(d) Observation plane $z=0.0$.

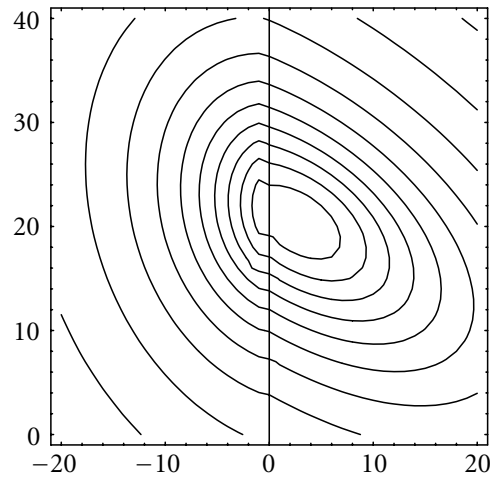

(f) Observation plane $z=30.0$.

Figure 5.4. Tensors $\sigma^{2 a}$ and $\sigma^{2 b}$ vertical and horizontal observation plane equipotential contours for the mise-à-la-masse potential arising from a conductive body. 


\section{Discussion and conclusions}

An integral equation is presented for the potential arising from a direct current point source in an anisotropic half-space with a vertical contact, containing a 3D conductive body. To solve this integral equation efficiently, the method of subsections, which is based on the numerical solution of this integral equation, has been used. If we choose the surface elements of the body (cell surfaces) to be parallel to the coordinate planes, the subarea integral can be calculated using a one-dimensional analytical method followed by another multidimensional numerical technique (Li and Uren, 1997).

Using this approach, highly accurate results can be obtained as shown in the numerical examples. The cube-cell accumulation method can handle the problem of calculating anomalies due to arbitrarily shaped bodies. We have shown, using the concept of induced surface charge density, how body and current locations as well as the nature of the vertical contact and anisotropic conductivity tensors affect the equipotential contours arising from a current source in a conductive body.

The results show that a vertical contact in an anisotropic space seriously affects the potential field. This indicates that field data is frequently disturbed by vertical discontinuities. The distance between the body and vertical contact is a major controlling factor of the effect.

\section{References}

[1] M. W. Asten, The influence of electrical anisotropy on mise-à-la-masse surveys, Geophys. Prosp. 22 (1974), no. 2, 238-245.

[2] E. H. Eloranta, The modelling of mise-à-masse anomalies in an anisotropic half-space by the integral equation method, Geoexploration 25 (1988), 93-101.

[3] L. Eskola, Addendum to "the solution of the stationary electric field strength and potential of a point current source in a $2 \frac{1}{2}$-dimensional environment" by L. Eskola and H. Hongisto, Geophys. Prosp. 32 (1984), 510-511.

[4] , Reflections on the electrostatic characteristics of direct current in an anisotropic medium, Geoexploration 25 (1988), no. 3, 211-217.

[5]_ Geophysical Interpretation Using Integral Equations, Chapman \& Hall, London, 1992.

[6] L. Eskola and H. Hongisto, The solution of the stationary electric field strength and potential of a point current source in a $2 \frac{1}{2}$-dimensional environment, Geophys. Prosp. 29 (1981), 249-266.

[7] _ Resistivity and IP modelling of an anisotropic body located in an isotropic environment, Geophys. Prosp. 45 (1997), no. 1, 127-139.

[8] L. Eskola, H. Soininen, and M. Oksama, Modelling of resistivity and IP anomalies of a thin conductor with an integral equation, Geoexploration 26 (1989), no. 2, 95-104.

[9] M. J. Flykt, E. H. Eloranta, K. I. Nikoskinen, I. V. Lindell, and A. H. Sihvola, DC potential anomalies caused by a conducting body in an anisotropic conducting half-space, IEEE Trans. Geosci. Remote Sens. 34 (1996), no. 1, 27-32.

[10] P. Li and N. F. Uren, Analytical solution for direct current electric potential in an anisotropic half-space with a vertical contact, 67th Annual International Meeting of the Society of Exploration Geophysicists, Society of Exploration Geophysicists, Texas, 1997, pp. 410-414, expanded abstracts.

[11] - Analytical solution for the point source potential in an anisotropic 3-D half-space. I. Two-horizontal-layer case, Math. Comput. Modelling 26 (1997), no. 5, 9-27. 
[12] Analytical solution for the point source potential in an anisotropic 3-D half-space. II. With two-vertical boundary planes, Math. Comput. Modelling 26 (1997), no. 5, $29-52$.

[13] The modelling of direct current electric potential in an arbitrarily anisotropic half-space containing a conductive 3-D body, J. Appl. Geophys. 38 (1997), no. 1, 57-76.

[14] Analytical solution for the electric potential due to a point source in an arbitrarily anisotropic half-space, J. Engrg. Math. 33 (1998), no. 2, 129-140.

[15] J. G. Negi and P. D. Saraf, Anisotropy in Geoelectromagnetism, Elsevier Scientific Publishers, Amsterdam, 1989.

[16] B. P. Pal and S. P. Dasgupta, Electrical potential due to a point current source over an inhomogeneous anisotropic earth, Geophys. Prosp. 32 (1984), 943-954.

[17] B. P. Pal and K. Mukherjee, Electrical potential due to a point current source over a layered conducting earth with dipping anisotropy, Geoexploration 24 (1986), 15-19.

[18] L. A. Tabarovskii, Integral equation in anisotropic media, Sov. Geol. Geophys. 18 (1977), 65-71.

[19] _ Properties of the potential of a simple layer in an anisotropic medium, Sov. Geol. Geophys. 18 (1977), 67-73.

[20] Z. Xiong, Y. Luo, S. Wang, and G. Wu, Induced-polarization and electromagnetic modeling of a three-dimensional body buried in a two-layer anisotropic earth, Geophysics 51 (1986), no. 12, 2235-2246.

Ping Li: School of Ecology and Environment, Centre for Applied Dynamical Systems and Environmental Modelling, Deakin University, P.O. Box 423, Warrnambool, Victoria 3280, Australia

E-mail address: ping_6155@email.com

Frank Stagnitti: School of Ecology and Environment, Centre for Applied Dynamical Systems and Environmental Modelling, Deakin University, P.O. Box 423, Warrnambool, Victoria 3280, Australia

E-mail address: frankst@deakin.edu.au 


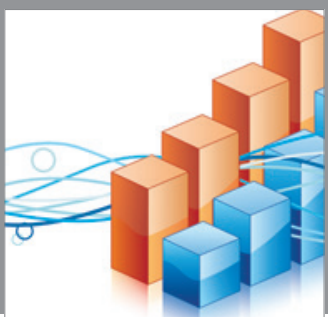

Advances in

Operations Research

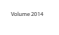

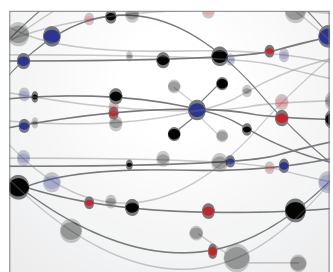

\section{The Scientific} World Journal
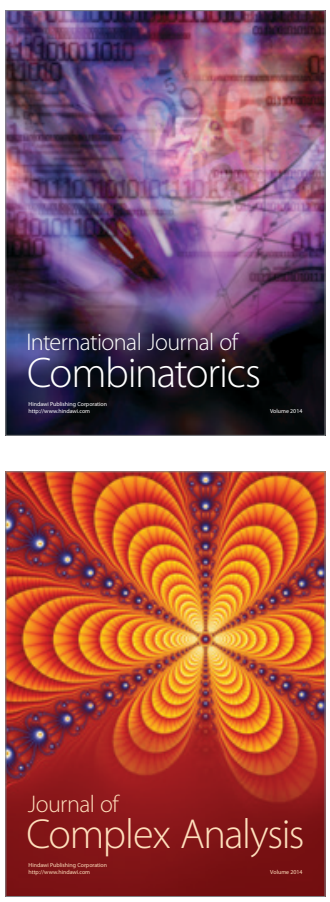

International Journal of

Mathematics and

Mathematical

Sciences
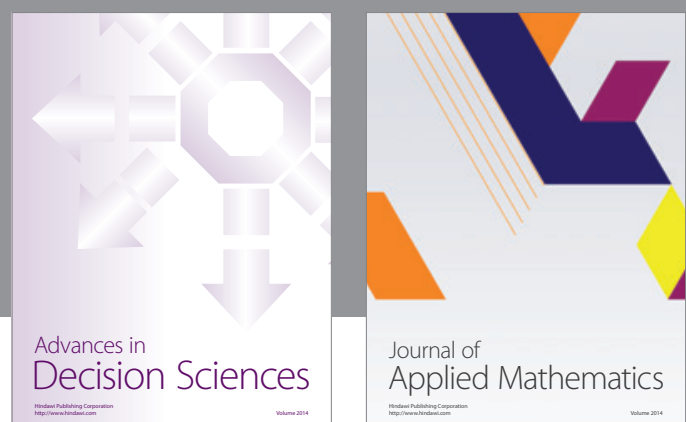

Journal of

Applied Mathematics
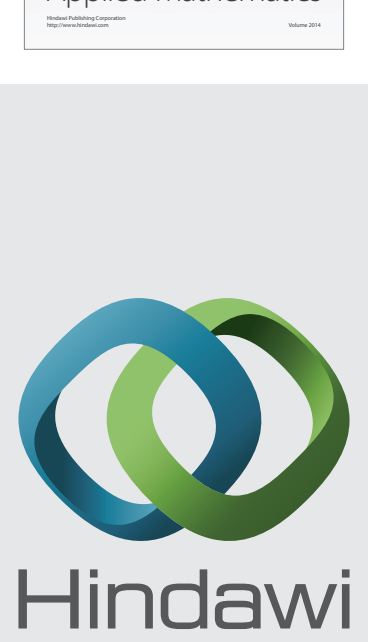

Submit your manuscripts at http://www.hindawi.com
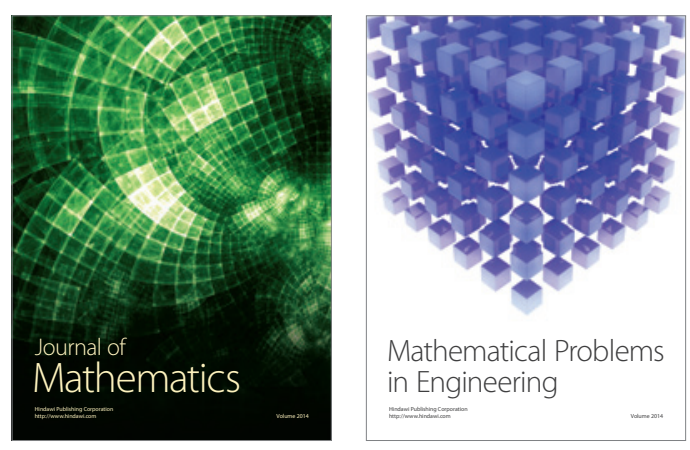

Mathematical Problems in Engineering
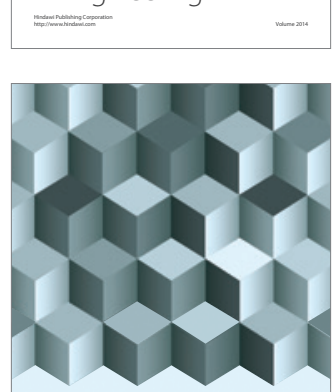

Journal of

Function Spaces
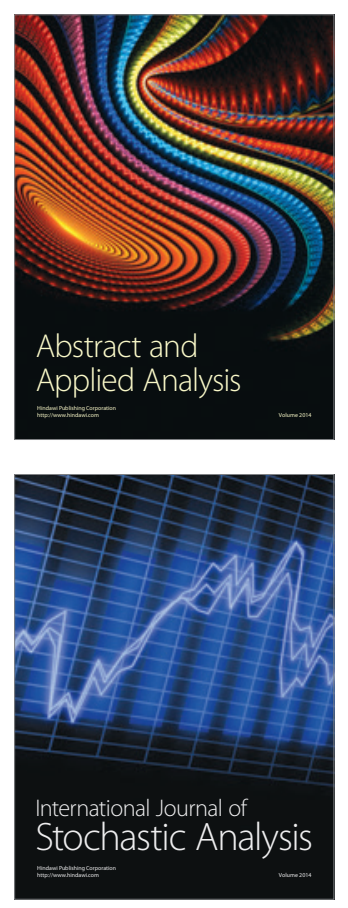

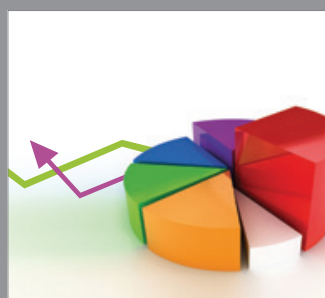

ournal of

Probability and Statistics

Promensencen
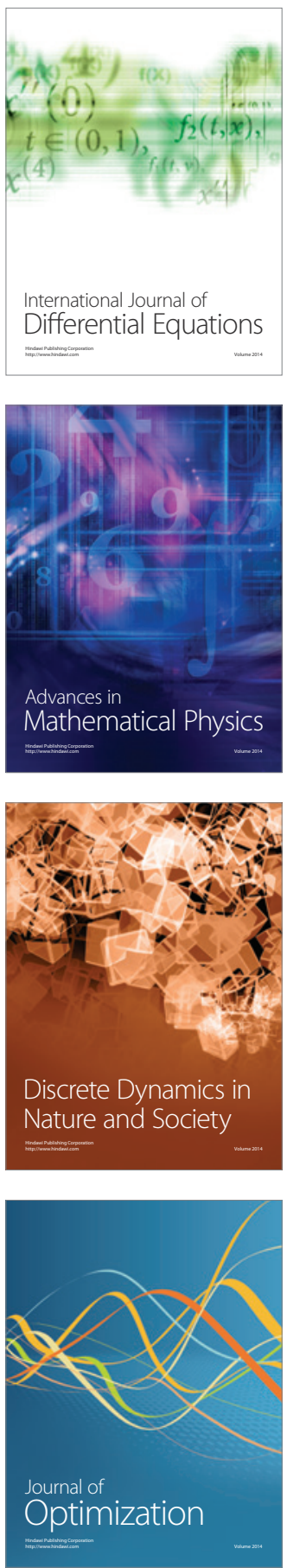